\title{
Resolved Schmidt-Kennicutt Relation for Star Forming Regions in the Galaxy and Magellanic Clouds
}

\author{
C.-H. R. Chen ${ }^{1}$, R. Indebetouw ${ }^{2}$, E. Muller ${ }^{3}$, M. Messineo ${ }^{1}$, \\ K. M. Menten ${ }^{1}$ and the SAGE-SMC Team \\ ${ }^{1}$ Max-Planck-Institut für Radioastronomie, D-53121 Bonn, Germany, email: rchen@mpifr.de; \\ ${ }^{2}$ University of Virginia/NRAO, Charlottesville, VA22904, USA; \\ ${ }^{3}$ National Astronomical Observatory of Japan, Mitaka, Tokyo 181-8588, Japan
}

\begin{abstract}
The relationship between star formation rate (SFR) and the gas surface density $\left(\Sigma_{\text {gas }}\right)$ is one of the most critical links between star formation and galaxy evolution. The observed SFR- $\Sigma_{\text {gas }}$ relation, the "Schmidt-Kennicutt (S-K) law", is tight when properties are averaged over kpc, but breaks down at the scale of giant molecular clouds (GMCs). To understand the physics governing the variations at GMC scales and the tight correlation at kpc scales, spatially and temporally resolved data covering a wide range of linear scale are needed. We have used the Spitzer surveys of the Large Magellanic Cloud and Magellanic Bridge to identify massive young stellar objects (YSOs), estimate "instantaneous" SFRs, and compare them to the S-K relation. These instantaneous SFRs are further compared to that estimated from integrated $\mathrm{H} \alpha$ and $24 \mu \mathrm{m}$ luminosities to examine how SFRs vary on 10 Myr timescales. We have also used SINFONI near-IR integral field spectra of two Galactic mini-starbursts W31 and W43 to determine their underlying massive stellar content, estimate the SFRs, and compare to the S-K relation. To investigate evironmental effects on star formation, we have used complete YSO samples in the LMC and the Bridge to estimate global star formation efficiencies (SFE) in these two systems.
\end{abstract}

Keywords. stars: formation — stars: early-type - HII regions - Galaxy: general — Magellanic Clouds

\section{Introduction}

Star formation is one of the fundamental processes in galaxy evolution. As stars form from interstellar gas, it is of critical importance to study and understand the relationship between star formation rate (SFR) and physical properties of interstellar gas. In extragalactic studies, it is possible to obtain global views of star formation, but the limited spatial resolution prohibits detailed examination of this physical process. Consequently, only the relationship between SFR and the surface density of interstellar gas $\left(\Sigma_{\text {gas }}\right)$ has been determined in these studies. The most widely cited SFR $-\Sigma_{\text {gas }}$ relationship is the Schmidt-Kennicutt (S-K) relation (Kennicutt 1989). The S-K relation can be simply expressed as $\Sigma_{\mathrm{SFR}} \propto\left(\Sigma_{\text {gas }}\right)^{N}$, with index $N=1.0-1.4$, depending on the galaxy sample used in the study. Furthermore, the S-K relation is valid only on large scales, as it appears to break down at scales $\leqslant 0.3 \mathrm{kpc}$ (Schruba et al. 2010; Onodera et al. 2010).

To understand these deviations from the S-K relation, one needs to look into the star formation tracers based on which the SFR is derived. The $\mathrm{H} \alpha$ and dust emission traces $\mathrm{O}$ stars with ionizing powers; thus, SFR derived from $\mathrm{H} \alpha+24 \mu \mathrm{m}$ luminosities is sensitive to star formation over the past $\sim 10 \mathrm{Myr}$ (Calzetti et al. 2007). The lifetime of a giant molecular cloud (GMC), on the other hand, has been estimated to be $<10$ to $\sim 30 \mathrm{Myr}$, depending on assumptions and methods used in the derivation (Kawamura et al. 2009; 
Blitz et al. 2007). It is conceivable that the SFR and GMC content averaged over too small a surface area do not adequately sample GMCs at different evolutionary stages, and thus do not show a good correlation. On the other hand, the SFR of a GMC also depends on the star formation efficiency (SFE). It has been observed that the SFE is lower in the outer disks of galaxies (Bigiel et al. 2010). It is understandable that the $\mathrm{SFR}-\Sigma_{\text {gas }}$ relationship in regions with low SFE deviates from the S-K relation.

\section{The Galaxy and the Magellanic Clouds as the Laboratory}

To understand the physics governing the variations at GMC scales and the tight correlation at kpc scales, spatially and temporally resolved data covering a wide range of linear scales are needed. The Galaxy provides the most detailed information. In the GLOSTAR program that aims to obtain a global view of star formation in the Milky Way, we have made an APEX Telescope Large Area Survey of the Galaxy (ATLASGAL) mapping of the detailed structures of GMCs, conducted the Bar and Spiral Structure Legacy Survey (BeSSeL) using masers to determine distances to GMCs, and carried out a successful near-IR spectroscopic survey of obscured massive stars and clusters. The Large and Small Magellanic Clouds (LMC and SMC) and the Magellanic Bridge (hereafter the Bridge), close extragalactic systems that have low metallicities of $1 / 3$, $1 / 5,1 / 8 Z_{\odot}$, respectively, provide environments different from the Galaxy to examine the environmental effects on SFEs. Recent Spitzer Legacy Surveys of the LMC and SMC, SAGE-LMC and SAGE-SMC, made it possible to obtain a global census of massive young stellar objects (MYSOs), compare them to the interstellar environment, and determine the "instantaneous" SFRs of GMCs.

\section{Resolved S-K Relation in Local Star Forming Regions}

To critically examine the SFR- $\Sigma_{\text {gas }}$ relationship, we have selected star forming regions W31 and W43 in the Galaxy, N44 and N159 in the LMC, and the high N(HI) part of the Bridge to study their massive star and YSO contents so that they can be compared with the associated GMCs to investigate the star formation process in detail (Chen et al. 2010, 2012). In each region in the LMC and the Bridge, the SFRs are estimated with different tracers to probe different timescales of star formation: the SFR determined from $\mathrm{H} \alpha+24 \mu m, \Sigma_{H \alpha+24}$, and that determined from YSOs, $\Sigma_{\mathrm{YSO}}$.

As shown in Fig. 1, the Bridge has expected SFR from $\Sigma_{\text {gas }}, \Sigma_{\mathrm{SFR}, \text { gas }} \sim 31 \times \Sigma_{H \alpha+24}$, while $\Sigma_{\mathrm{YSO}}$ is in agreement with $\Sigma_{\mathrm{SFR} \text {,gas }}$ within a factor of 2 . Compared to our studies on GMCs in the LMC (Indebetouw et al. 2008; Chen et al. 2010), the Bridge shows a consistent trend with GMCs without bright HII regions (i.e., low star formation activities). We attributed this lower value of $\Sigma_{H \alpha+24}$ to the lower luminosity to mass (L/M) ratios in such GMCs in the LMC, as star formation occurred mostly in lower-mass or less rich clusters that do not fully sample the high-mass end of the stellar initial mass function and thus have a lower L/M ratio than rich clusters analyzed in Calzetti et al. (2007). Fig. 1 also shows that for GMCs associated with bright HII regions in the LMC, both $\Sigma_{\mathrm{YSO}}$ and $\Sigma_{H \alpha+24}$ are in agreement with $\Sigma_{\mathrm{SFR} \text {,gas }}$. However, the ratios of current-to-past SFRs, $\Sigma_{\mathrm{YSO}} / \Sigma_{H \alpha+24}$ of these regions range from 0.4 to 2.1 . These ratios suggest that the current SFR may be lower or higher than the last $10 \mathrm{Myr}$, not constant over time. Hence the assumption of constant SFR commonly used in star formation studies needs to be reconsidered.

In each starburst region in the Galaxy, the SFRs are estimated both with the resolved OB stellar content and with integrated $8 \mu \mathrm{m}$ luminosities. Fig. 1 shows that the former are 


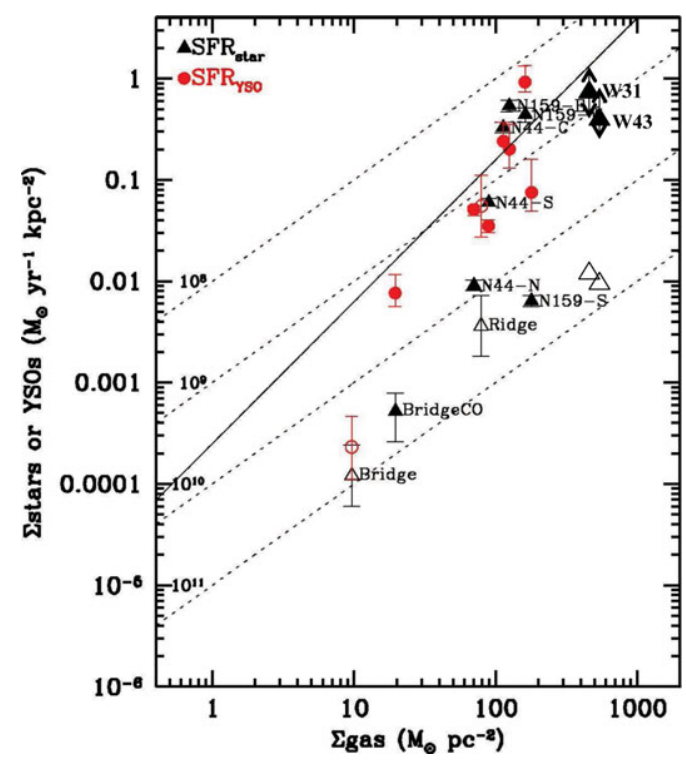

Figure 1. Relation between the SFR per unit area and $\Sigma_{\text {gas }}$ of molecular clouds in the Bridge (filled symbols: all molecular clouds; open symbols: the entire Bridge area) and selected star forming regions in the LMC (N44-C,S,N and N159-E,W,S) and the Galaxy (W31 and W43) (Chen et al. 2010, 2012). Each region has two estimated SFRs using different tracers. In the LMC and the Bridge, $\Sigma_{\text {star }}$ (filled triangles) and $\Sigma_{\mathrm{YSO}}$ (filled circles) are estimated from $L_{H \alpha+24 \mu m}$ and resolved YSO content, respectively. In the Galaxy, $\Sigma_{\text {star }}$ are estimated from $L_{8 \mu \mathrm{m}}$ (open triangles) and resolved massive stellar content (filled triangles), respectively. The solid line is the S-K relation, and the dotted lines correspond to gas depletion timescales at constant SFRs from $10^{8}$ to $10^{11} \mathrm{yr}$.

in good agreement with $\Sigma_{\mathrm{SFR}, \text { gas }}$, while $\Sigma_{8 \mu m}$ is $>10$ times smaller. Since $8 \mu m$ emission mostly originates from small dust grains that can be easily destroyed under the intense radiation field in starbursts, SFR tracers need to be carefully selected for such regions.

\section{Environmental Effects on Star Formation}

To investigate the environmental effects such as metallicity or galactic environment on star formation, we examine $\epsilon$, the fraction of HI resolution elements containing YSOs as a function of $\mathrm{N}(\mathrm{HI})$ in the entire LMC and the Bridge. $\epsilon$ is equivalent to the probability of finding YSOs at a given N(HI). Fig. 2 shows the histograms of the LMC's and the Bridge's HI resolution elements, i.e., 30" pixel $^{-1}$ (Kim et al. 2003; Muller et al. 2003), and YSOs, as well as $\epsilon$ as a function of $\mathrm{N}(\mathrm{HI})$. Comparisons of $\epsilon$ show that the Bridge is $\lesssim 1 / 3$ the LMC in their overlapping bins of $\mathrm{N}(\mathrm{HI})=12-24 \times 10^{20} \mathrm{~cm}^{-2}$. However, at lower $\mathrm{N}(\mathrm{HI})$, the Bridge shows a flatter slope, with $\epsilon$ up to $\sim 2$ times the LMC.

To investigate the possible causes of different $\epsilon$ in the LMC and the Bridge, we examine the YSO properties in these two systems. The YSOs in the Bridge have inferred masses of 4-10 $M_{\odot}$, not as massive as those of 4-45 $M_{\odot}$ found in the LMC (Chen et al. 2009, 2010, 2012). Furthermore, Bridge YSOs are sparsely distributed, in sharp contrast to LMC YSOs that are usually in groups and clusters. The lack of massive clusters in the Bridge is consistent with the simulations of Krumholz et al. (2009), in which cluster-forming molecular clouds are scarce at that density and metallicity. On the other hand, the reverse trend appears to suggest a different dominant mechanism of star formation at the lower $\mathrm{N}(\mathrm{HI})$ regime. The sparse distribution of YSOs and low masses of molecular clouds, $10^{3}$ $10^{4} M_{\odot}$, in the Bridge (Mizuno et al. 2006), as well as the rapid star formation implied by the tight spatial correlation between molecular clouds and YSOs, are consistent with star formation through colliding flows (Heitsch et al. 2006). The comparisons in $\epsilon$ and star formation properties between the LMC and the Bridge indicate that at the higher $\mathrm{N}(\mathrm{HI})$ regime, the lower metallicity of the Bridge is likely responsible for lower efficiencies 

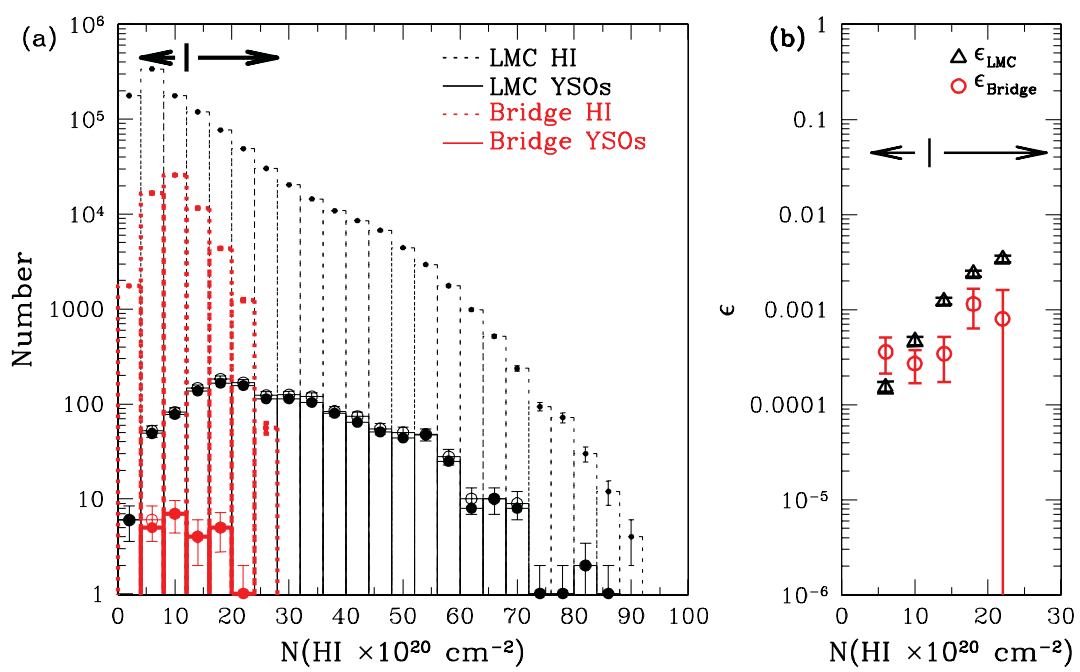

Figure 2. (a) Histograms of all HI resolution elements in the LMC (black dotted lines) and those containing YSOs (black solid lines, Gruendl \& Chu 2009). Similar histograms for the Bridge are shown as red dotted and solid lines. (b) $\epsilon$ as a function of $\mathrm{N}(\mathrm{HI})$ for the LMC (black triangles) and the Bridge (red circles). Error bars are marked. The Bridge has $\epsilon \sim 1 / 3$ the LMC at N(HI) $\geqslant 12 \times 10^{20} \mathrm{~cm}^{-1}$. However, at lower N(HI), the Bridge's $\epsilon$ is comparable or even larger than the LMC. Figure taken from Chen et al. (2012).

of star and molecular cloud formation. At the lower N(HI) regime, the Bridge's dynamic tidal environment may enhance distributed star formation.

\section{Acknowledgements}

We thank the SOC and LOC for organizing such a stimulating meeting. We acknowledge the financial support by the European Research Council for the ERC Advanced Grant GLOSTAR under constract no. 247078.

\section{References}

Bigiel, F., Leroy, A., Walter, F., et al. 2010, AJ, 140, 1194

Blitz, L., Fukui, Y., Kawamura, A., et al. 2007, Protostars and Planets V, 81

Calzetti, D., Kennicutt, R. C., Engelbracht, C. W., et al. 2007, ApJ, 666, 870

Chen, C.-H. R., Chu, Y.-H., Gruendl, R. A., Gordon, K. D., \& Heitsch, F. 2009, ApJ, 695, 511

Chen, C.-H. R., Indebetouw, R., Chu, Y.-H., et al. 2010, ApJ, 721, 1206

Chen, C.-H. R., Indebetouw, R., Muller, E., et al. 2012, ApJ, submitted

Gruendl, R. A. \& Chu, Y. 2009, ApJS, 184, 172

Heitsch, F., Slyz, A. D., Devriendt, J. E. G., Hartmann, L. W., \& Burkert, A. 2006, ApJ, 1052 Indebetouw, R., Whitney, B. A., Kawamura, A., et al. 2008, AJ, 136, 1442

Kawamura, A., Mizuno, Y., Minamidani, T., et al. 2009, ApJS, 184, 1

Kennicutt, R. C., Jr. 1984, ApJ, 287, 116

- 1989, ApJ, 344, 685

Kim, S., Staveley-Smith, L., Dopita, M. A., Sault, R. J., Freeman, K. C., Lee, Y., \& Chu, Y. 2003, ApJS, 148, 473

Krumholz, M. R., McKee, C. F., \& Tumlinson, J. 2009, ApJ, 693, 216

Mizuno, N., Muller, E., Maeda, H., et al. 2006, ApJL, 643, L107

Muller, E., Staveley-Smith, L., Zealey, W., \& Stanimirović, S. 2003, MNRAS, 339, 105

Onodera, S., Kuno, N., Tosaki, T., et al. 2010, ApJL, 722, L127

Schruba, A., Leroy, A. K., Walter, F., et al. 2010, ApJ, 722, 1699 